\title{
Traffic State Recognition of Intersection Based on Image Model and PCA Hashing
}

\author{
Li-li Zhang $\mathbb{D})^{1,2}$ Li Wang $\mathbb{D}^{2}{ }^{2}$ and Qi Zhao $\mathbb{D}^{2}$ \\ ${ }^{1}$ College of Information Engineering, Beijing Institute of Petrochemical Technology, Beijing 102617, China \\ ${ }^{2}$ Beijing Key Lab of Urban Intelligent Control Technology, North China University of Technology, Beijing 100144, China \\ Correspondence should be addressed to Li-li Zhang; zllphd2012@163.com
}

Received 19 August 2019; Revised 20 January 2020; Accepted 17 February 2020; Published 15 June 2020

Academic Editor: Shamsunnahar Yasmin

Copyright (c) 2020 Li-li Zhang et al. This is an open access article distributed under the Creative Commons Attribution License, which permits unrestricted use, distribution, and reproduction in any medium, provided the original work is properly cited.

The premise of implementing an effective traffic control strategy is the accurate traffic state recognition. In the existing study, traffic state recognition methods were processed by using statistical characteristics and long-term scale detection of field traffic data. Hence, the dynamic characteristics and subtle changes in traffic flow were easy to overlook. At present, more and more advanced traffic detection technology provides reliable and accurate data for measuring and distinguishing the state of urban road traffic, such as the cooperative vehicle-infrastructure system, wide-area radar technology, and 5G technology. This study proposes a novel method called HTSI (High Precision Traffic State Identification Method), which is based on the advanced detection technology in traffic state recognition at the intersection: The raw data used for intersection traffic state recognition is highprecision detection data of tracking characteristics, which make the data look like a picture of the intersection at God's perspective. To this end, we construct an image model for intersections and implement image feature extraction in a way that is different from traditional image processing. Then, the traffic state recognition problem at the intersection is translated into an image searching problem with tags. The image searching is realized by the hashing algorithm. Finally, the comprehensive experiments prove that the proposed method is more accurate and finer than other methods.

\section{Introduction}

Intersections are important nodes of urban road networks, and traffic demand often gathers at intersections. The multidimensional, complex, and time-varying characteristics of traffic demand would reflect on traffic states at intersections. Therefore, the premise of implementing effective traffic control and preventing traffic congestion is that the accurate traffic states recognition at the intersection are prepared for recognition.

Nowadays, the most widely used traffic signal control systems such as SCOOT [1,2] and SCATS [3] need to accurately identify traffic conditions to ensure that the control strategy being implemented is effective. However, due to the limitations of the existing detection techniques and types of detection data, the accuracy of traffic state recognition of these systems is not sufficient. Specifically, the existing detection technology is mainly based on cross-sectional detection, and the output data are mainly statistical data. Although these data can reflect the movement of some traffic flows, the details are often ignored.

Recently, with the development of the cooperative vehicle-infrastructure system $[4,5]$, wide-area radar technology $[6,7]$, and 5G technology [8-10], the low-latency, highprecision, and traceable techniques have been providing new ideas for the recognition of intersection traffic conditions, especially in the accuracy of recognition and the restoration of traffic flow details. Surprisingly, new technologies have been widely used in traffic control $[11,12]$, traffic guidance $[13,14]$, and autonomous driving [15-17], but are not used for traffic state recognition.

If we consider the traffic flow at an urban intersection as a video, then the video is composed of images of $n$ frames. If we assume that the detector at the intersection is the lens of this camera, then the acquisition process of the detector is the process of recording the video. The frequency of the 
detector is the camera's shooting frequency. Based on the abovementioned assumptions, we can map the data of the detector into image data. This assumption would be realized by the proposed HTSI method rather than the existing method. In this way, the research results in the field of image processing can be introduced into the study of traffic state recognition at intersections. Then, the traffic problem can be converted into an understanding problem of the image content $[18,19]$ or image search problem [20-22].

The main contributions of this paper include the following:

(i) We propose an intersection image model based on extended discrete state coding using raw data with high-precision detection.

(ii) We convert the problem of the recognition of traffic conditions at intersections into the problem of searching car images, which was first proposed in the field. This makes it possible to use a variety of sophisticated algorithms in the field of image search.

(iii) We further demonstrate the superiority of this method. Specifically, it has advantages over the existing methods in terms of the fineness and effectiveness of traffic state recognition.

The remainder of this paper is organized as follows: Section II presents a literature review of related work. Section III presents the detail of our image model of intersection and image feature extraction. Section IV describes the transformation of the traffic state recognition problem at the intersection into an image search problem and designs an image search based on PCA (Principal Components Analysis, PCA) hashing. Section V describes the simulation experiments and presents the experimental results. Section VI concludes this paper and discusses directions for future research.

\section{Related Work}

The traffic state recognition at urban road intersections is an ancient problem that dates back to the 1950s [23]. It is also a very important issue of urban road traffic control because only effective traffic state recognition can implement effective traffic control strategies. So, it has attracted many scholars to study, and existing methods can be generally categorized into two classes.

\subsection{Traffic State Recognition Based on Queue Length} Estimation. To estimate the queue length in each direction of the intersection with single detector data [24-30] or multiple source-detector data [31,32] or vehicle road collaboration data [33], the queue length is used as the basis for traffic state recognition. It can be judged through checking whether the queue length reaches the threshold.

\subsection{Traffic State Recognition Based on Traffic Parameters.} Delays [34] and time occupancy [35-38] are also commonly used as parameters for traffic state recognition at intersections. The traditional detector can effectively measure the delay and time occupancy and can reflect the motion state of the vehicle through these two parameters. This type of model can be used to determine the traffic state at the intersection.

However, to the best of our knowledge, the abovementioned two methods are difficult to obtain satisfactory results in the fineness and effectiveness of traffic state recognition at intersections. The main reason is that these methods are limited by traditional detection techniques. The traditional detection data have the characteristics of coarse granularity and strong statistical properties. They ignore the intricate details of traffic flow in nature. For example, it is sometimes wrong to treat a traffic accident (As shown in Figure 1(b)) near an intersection as a state of oversaturation (as shown in Figure 1(a)), but this is not the case.

\section{Proposed Method}

3.1. Problem Statement. In this paper, we consider a typical signalized urban road intersection. The vehicles usually gather at intersections because the intersection will separate the traffic demand in time and space to ensure safety. The characteristics of traffic demand and the traffic flow are dynamic and time-varying. So, it is difficult to identify these changes sensitively and accurately using the existing traffic detection techniques, but using advanced detection techniques such as road coordination and wide-area radar can obtain more accurate and time-series finer raw data. Suppose we consider the process of traffic detection as taking pictures. We can get pictures with continuous time series (as shown in Figure 2) by increasing the frequency of detection, just like the continuous observation of changes in traffic demand with God's perspective (as shown in Figure 3). This assumption is very interesting and meaningful.

\subsection{Image Model of Intersection}

3.2.1. Raw Data for Advanced Detection Methods. Traditional traffic detection data for traffic demand measurement include traffic, occupancy, density, and queue length. These types of detection data have the characteristics of detecting coarse granularity and strong statistical properties. It is difficult to effectively describe the details of realtime dynamic traffic using them. Advanced detection technology represented by a cooperative vehicle-infrastructure system and wide-area radar technology has developed rapidly, which makes real-time high-precision trajectory detection possible. It is gradually applied to the field of urban road traffic detection based on these advanced technologies (as shown in Figure 4).

These advanced detection techniques generally provide real-time data in milliseconds. Data types include instantaneous vehicle velocity, vehicle latitude and longitude, vehicle ID, vehicle length, and time stamp (as shown in Table 1 for an example of raw data for a wide-area radar detector.). At the same time, they can provide full sample data with a small error within the effective detection range. The true appearance of the traffic demand can be fully 


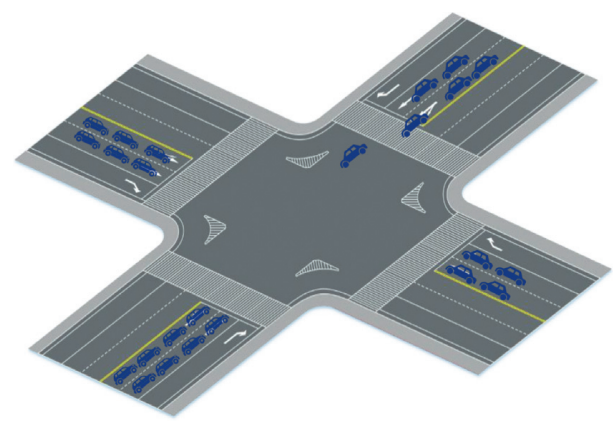

(a)

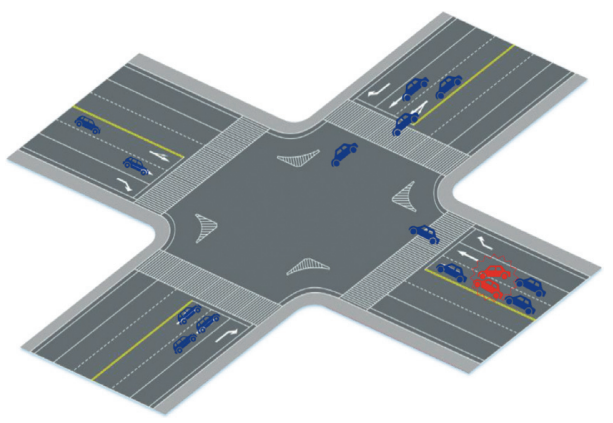

(b)

Figure 1: Oversaturation caused by different reasons. (a) Oversaturation of intersections due to large traffic demand and (b) oversaturation of intersections due to a traffic accident.
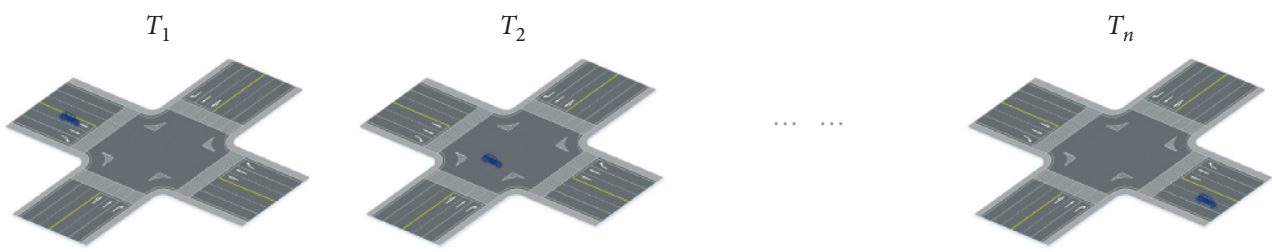

FIgURE 2: Continuous time series picture.

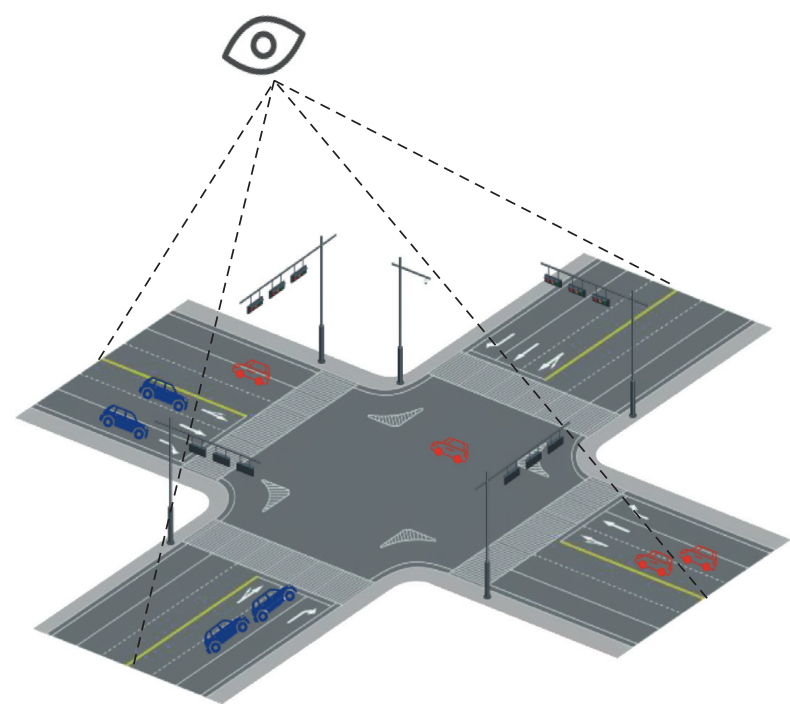

FIGURE 3: God's perspective.

reflected when using these full-sample real-time high-precision trajectory data because these data are very subtle and sensitive.

3.2.2. Extended Discrete State Coding. A full-color image is composed by red-green-blue (RGB) color. Inspired by this, we develop a five-tuple extended discrete state coding $[39,40]$ method. It is used to indicate the attributes of the vehicle and the operation of the vehicle in the effective detection area at the intersection. This quintuple corresponds to the five data types given in Table 1 .

$$
M \in(\mathbb{C} \times \mathbb{R} \times \mathbb{Z} \times \mathbb{Q} \times \mathbb{N})^{L_{P} / l} .
$$

The effective detection area of the intersection is $L_{p} * L_{p}$, which it is determined by the actual detection distance of the detector. However, it should be emphasized that the boundary of the effective detection area should be larger than the maximum queue at intersection when it has reached oversaturation. The lane can be divided into $L_{p} / l$ units with the equivalent car length $l$ as a standard. $M$ represents the attributes and operation of the vehicle in the effective detection area at intersection after the discretization. $\mathbb{C}$ denotes whether the lane unit would store information in the presence of the vehicle (one indicates the presence of the vehicle; Zero indicates no vehicle. This description is the result of mapping the real coordinate data to the road segment). $\mathbb{R}$ denotes speed information of the stored vehicle (one indicates that the vehicle speed is free-flowing. Zero means no car or vehicle stopped. The rest of the values are obtained by mapping the Sigmod function and retaining one decimal place.). $\mathbb{Z}$ denotes acceleration information of the stored vehicle (a negative number indicates the deceleration motion of the vehicle. Zero indicates the uniform motion of the vehicle. A positive number indicates the acceleration motion of the vehicle.). $\mathbb{Q}$ denotes identity information of the stored vehicle (the identification of the vehicle is numbered sequentially starting from the innermost lane.). $\mathbb{N}$ denotes lane change information of the stored vehicle (zero indicates that the vehicle has never changed lanes. One indicates the position of the vehicle after the lane change. 0.5 refers to the process of vehicle lane change.).

Figure 3(a) is the discrete state of the classic intersection at time $t$. The effective detection area of such an intersection can be represented by a quintuple. We convert it into an 

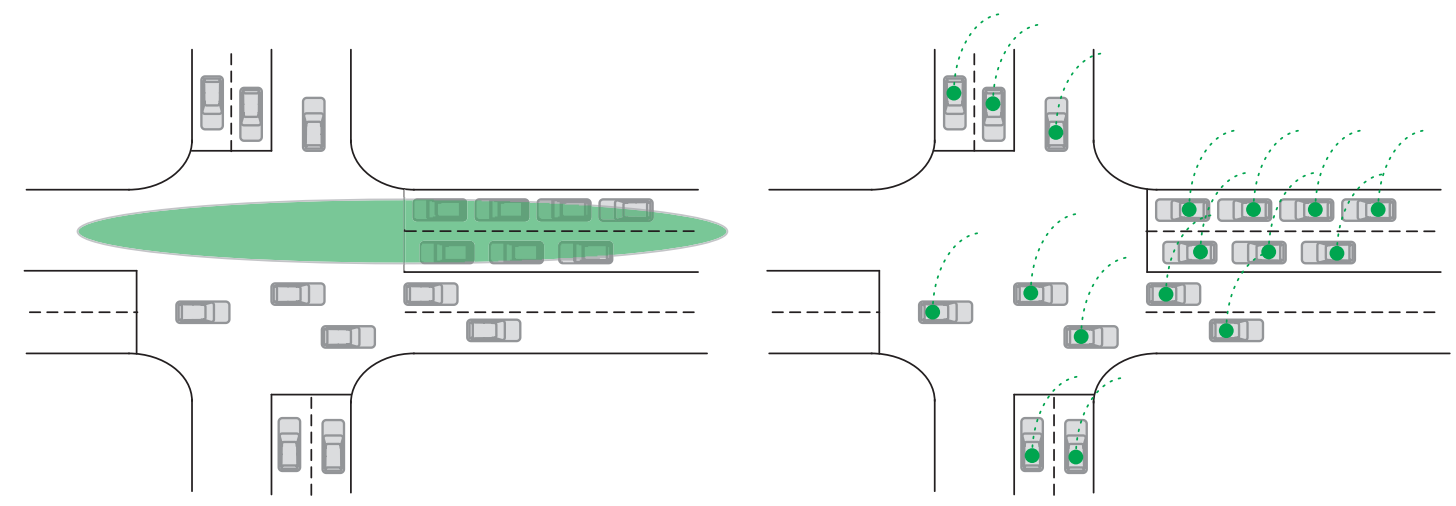

FIGURE 4: Real-time high-precision trajectory detection.

TABLE 1: Example of raw data for a wide-area radar detector.

\begin{tabular}{lccccc}
\hline \multirow{2}{*}{ Num } & & \multicolumn{2}{c}{ Type } & & \\
& Real-time speed: XSpeed, YSpeed & Latitude and longitude: XPos, YPos & ID & Vehicle length (m) & Time stamp \\
\hline 1 & $(-14.7500,-0.2500)$ & $(20.0011,3.7004)$ & 1 & 4.800000 & $2018-11-17$ 13:57:20.265 \\
2 & $(-8.5000,0.2500)$ & $(-5.2001,15.2994)$ & 2 & 4.800000 & $2018-11-17$ 13:57:20.265 \\
\hline
\end{tabular}

image consisting of "pixels," as shown in Figure 5(b). This has enabled the imaging modeling at the intersection.

3.3. Feature Extraction of Images at Intersections. The actual content of the image is determined by its characteristics, and the image of the intersection is no exception. Therefore, feature extraction of the image of the intersection is required. This section will give a detailed description of the method of feature extraction.

Assume that the effective detection area of the intersection is a set $\Gamma$ consisting of key parts.

$$
\Gamma=\{X, Y, Z\}
$$

where $X=\left\{x_{1}, x_{2}, \ldots, x_{m}\right\}$ represents the set of intersection entrance lanes and $m$ is the number of entrance lanes. $Y=$ $\left\{y_{1}, y_{2}, \ldots, y_{n}\right\}$ represents the set of intersection exit lanes, and $n$ is the number of exit lanes. $Z=\left\{z_{1}, z_{2}, \ldots, z_{u}\right\}$ represents a set of intersection conflict zones, and $u$ is a block of conflict zones. $X, Y$, and $Z$ are described as key parts of the effective detection area of the intersection, as shown in Figure 6.

As in equation (3), $\Gamma$ is calculated from the quintuple:

$$
e_{\eta \in \Gamma}(t)=\sum_{i=1, j=1}^{5} w_{i} \mathrm{M}_{j}=w_{1} \cdot \mathbb{C}(t)+w_{2} \cdot \mathbb{R}(t)+w_{3} \cdot \mathbb{Z}(t)
$$

$\mathbb{N}(t)$, s.t. $M_{j} \in\left[(\mathbb{C} \times \mathbb{R} \times \mathbb{Z} \times \mathbb{Q} \times \mathbb{N})^{L_{P} / l}\right], w_{4} \cdot \mathbb{Q}(t)+w_{5}$. where $\sum_{i=1}^{5} w_{i}=1,0<w_{i} \leq 1$ is the weight of the index.

The distance entropy [41] of the translation correction process is used to determine $e_{\eta \in \Gamma}(t)$.

Here, subindex $A=\left[\begin{array}{c}\bar{M}_{j}(t) \mid \bar{M}_{1}(t) \in \mathbb{C}(t), \bar{M}_{2}(t) \\ \in \mathbb{R}(t), M_{3}(t) \in \mathbb{Z}((t)), \bar{M}_{4}(t) \\ \in \mathbb{Q}(t), \bar{M}_{5}(t) \in \mathbb{N}(t), j=1,2,3,4,5\end{array}\right]$. Suppose that $\bar{M}_{j}=a_{j}$; there will be an information decision matrix
$A=\left[a_{j}\right]_{5}$, where $a_{j}$ is the observed value of the $j^{\text {th }}$ index, $i=1,2,3,4,5$.

(i) Step-1: the $A=\left[a_{j}\right]_{5}$ is normalized to obtain a standard information matrix $R=\left[r_{j}\right]_{5}$. The normalized approach is

For the larger and the better the subindex:

$$
r_{j}=\frac{a_{j}-\min \left(a_{j}\right)}{\max \left(a_{j}\right)-\min \left(a_{j}\right)} .
$$

For the smaller and the better the sub-index:

$$
r_{j}=\frac{\max \left(a_{j}\right)-a_{j}}{\max \left(a_{j}\right)-\min \left(a_{j}\right)}
$$

The optimal unit value $r_{j}^{*}$ corresponding to the $j^{\text {th }}$ indicator is selected, $j=1,2,3,4,5$. The rules are

$$
r_{j}^{*}=\left\{\begin{array}{l}
\max _{1 \leq j \leq 5}\left\{r_{j}\right\}, j \text { is benefit attribute } \\
\min _{1 \leq j \leq 5}\left\{r_{j}\right\}, j \text { is cost attribute }
\end{array}, \forall j\right.
$$

(ii) Step-2: the distance between $r_{j}$ and $r_{j}^{*}$ is calculated.

$$
d_{j}=\left|r_{j}-r_{j}^{*}\right|, \quad j=1,2,3,4,5
$$

(iii) Step-3: the probability of the index is calculated.

$$
\beta_{j}=\frac{\left(d_{j}+u_{j}\right)}{\sum_{j=1}^{5}\left(d_{j}+u_{j}\right)}, \quad j=1,2,3,4,5 .
$$




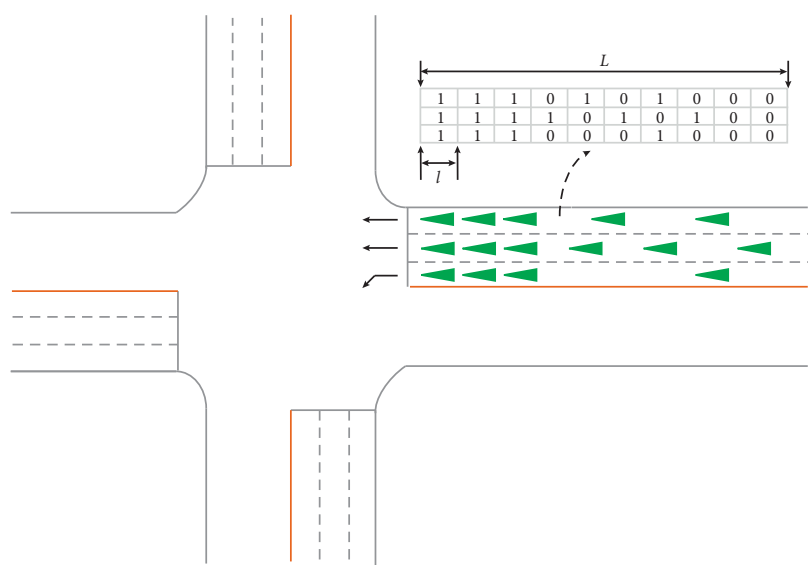

(a)

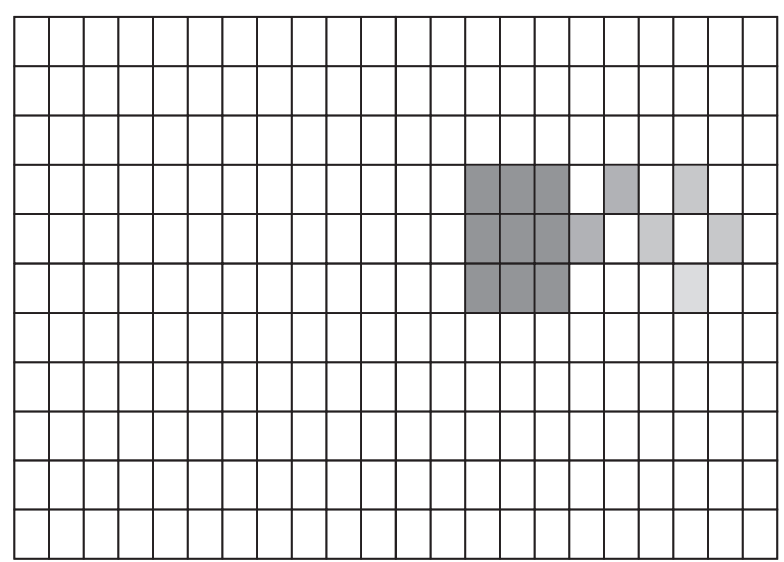

(b)

FIGURE 5: Extend discrete state coding to image mapping.

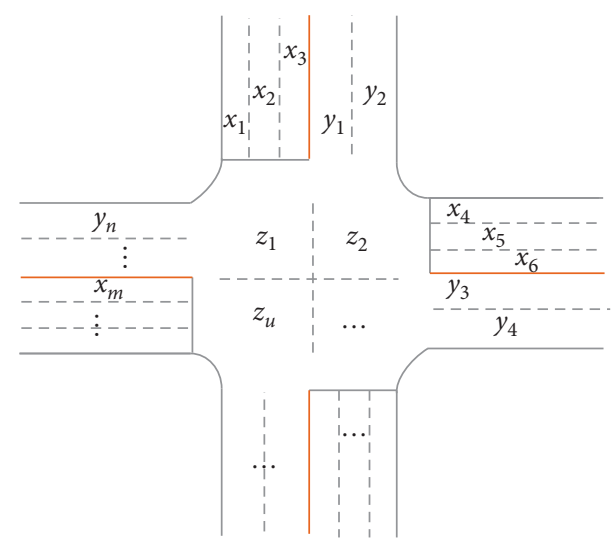

FIGURE 6: Combination of key parts at intersections.

When $d_{i}>0$, there was no need for revision to $\beta_{j}$, at that time $u_{i}=0$. When $d_{i}=0$, the index does not work to $e_{\eta \in \Gamma}(t)$, and in order to ensure the availability of all the index data, it needs human translation correction to $\beta_{j}$, this time, $u_{i}$ is constant and $u_{i}>0$.

(iv) Step-4: the distance entropy of the index is calculated.

$$
e_{j}=-\frac{1}{\ln 5} \sum_{j=1}^{5} \beta_{j} \ln \beta_{j}, \quad \forall j .
$$

(v) Step-5: the entropy weight is calculated.

$$
w_{i \propto j}=\frac{1-e_{j}}{5-\sum_{j=1}^{5} e_{j}}, \quad \forall j,
$$

where $\sum_{i=1}^{5} w_{i}=1,0<w_{i} \leq 1$.

(vi) Step-6: $e_{\eta \in \Gamma}(t)$ is obtained.

$$
e_{\eta \in \Gamma}(t)=\sum_{i=1, j=1}^{5} w_{i} \cdot M_{j}, \quad \forall i, j
$$

The characteristic values of the key parts of the intersection can be expressed as

$$
\begin{aligned}
& \left\{\begin{array}{l}
e_{x_{i} \in X}(t)=\left[e_{x_{1}}(t), e_{x_{2}}(t), \ldots, e_{x_{m}}(t)\right], \\
e_{y_{i} \in Y}(t)=\left[e_{y_{1}}(t), e_{y_{2}}(t), \ldots, e_{y_{n}}(t)\right], \\
e_{z_{i} \in Z}(t)=\left[e_{z_{1}}(t), e_{z_{2}}(t), \ldots, e_{z_{u}}(t)\right],
\end{array}\right. \\
& \text { s.t. } \quad e_{\eta \in \Gamma}(t)=\sum_{i=1, j=1}^{5} w_{i} \cdot \mathrm{M}_{j}, \quad \forall i, j .
\end{aligned}
$$

The traffic state of intersection approach is denoted as $S=\left[\bar{s}_{1}, \bar{s}_{2}\right]$. The traffic state of intersection departure lane is denoted as $S=\left[\bar{s}_{1}, \bar{s}_{2}\right]$. The traffic state of the conflict zone at the intersection is denoted as $S=\left[\bar{s}_{1}, \bar{s}_{2}\right]$, where $\bar{s}_{1}$ and $\bar{s}_{2}$, respectively, represent the undersaturated state and oversaturated state.

The piecewise linear function is designed as follows. It is used to describe the relationship between the traffic state and the characteristic value of each key part at the intersection.

$$
\left\{\begin{array}{l}
s_{e_{x_{i} \in X}}\left(x_{1}\right)= \begin{cases}e_{x_{1}}(t) \longrightarrow \bar{s}_{1}, & {\left[0, a_{1}\right),} \\
e_{x_{1}}(t) \longrightarrow \bar{s}_{2}, & \left(a_{1}, a_{2}\right],\end{cases} \\
s_{e_{y_{i} \in Y}}\left(x_{1}\right)= \begin{cases}e_{x_{1}}(t) \longrightarrow \bar{s}_{1}, & {\left[0, b_{1}\right),} \\
e_{x_{1}}(t) \longrightarrow \bar{s}_{2}, & \left(b_{1}, b_{2}\right],\end{cases} \\
s_{e_{z_{i} \in Z}}\left(x_{1}\right)= \begin{cases}e_{x_{1}}(t) \longrightarrow \bar{s}_{1}, & {\left[0, c_{1}\right),} \\
e_{x_{1}}(t) \longrightarrow \bar{s}_{2}, & \left(c_{1}, c_{2}\right] .\end{cases}
\end{array}\right.
$$

The piecewise linear function in equation (13) is vector transformed to obtain the following equation:

$$
\left\{\begin{array}{l}
s_{e_{x_{i} \in X}}\left(x_{1}\right)=\left[e_{x_{1}, \bar{s}_{1}}(t), e_{x_{1}, \bar{s}_{2}}(t)\right], \\
s_{e_{y_{i} \in Y}}\left(y_{1}\right)=\left[e_{y_{1}, \bar{s}_{1}}(t), e_{y_{1}, \bar{s}_{2}}(t)\right], \\
s_{e_{z_{i} \in Z}}\left(z_{1}\right)=\left[e_{z_{1}, \bar{s}_{1}}(t), e_{z_{1}, \bar{s}_{2}}(t)\right] .
\end{array}\right.
$$


Equation (15) is expressed as a set $\Xi$ of images of the traffic state of the intersection. $\xi_{i}$ represents the image in which the $i^{\text {th }}$ state is represented.

$$
\Xi=\left[\left|\xi_{1}, \xi_{2}, \ldots, \xi_{\omega}\right| \bowtie \geq 1\right]
$$

where $\xi_{i}$ is as shown in the following equation:

$$
\begin{aligned}
& \xi_{i}=\left[\begin{array}{lll}
s_{e_{x_{i} \in X}} & s_{e_{y_{i} \in Y}} & s_{e_{z_{i} \in Z}}
\end{array}\right]^{T} \\
& =\left[\begin{array}{llll}
s_{e_{x_{i} \in X}}\left(x_{1}\right) & s_{e_{x_{i} \in X}}\left(x_{2}\right) & \cdots & s_{e_{x_{i} \in X}}\left(x_{m}\right) \\
s_{e_{y_{i} \in Y}}\left(y_{1}\right) & s_{e_{y_{i} \in Y}}\left(y_{2}\right) & \cdots & s_{e_{y_{i} \in Y}}\left(y_{n}\right) \\
s_{e_{z_{i} \in Z}}\left(z_{1}\right) & s_{e_{z_{i} \in Z}}\left(z_{2}\right) & \cdots & s_{e_{z_{i} \in Z}}\left(z_{u}\right)
\end{array}\right] .
\end{aligned}
$$

Equation (16) is transformed as follows. Get $\sigma$ dimensional feature vector of the intersection image $\xi_{i}$.

$$
\begin{aligned}
& \overline{\xi_{i}}=\left[\begin{array}{lll}
s_{e_{x_{i} \in X}}^{T} & s_{e_{y_{i} \in Y}}^{T} & s_{e_{z_{i} \in Z}}^{T}
\end{array}\right]^{T}, \\
& =\left[\begin{array}{c}
s_{e_{x_{i} \in X}}\left(x_{1}\right) s_{e_{x_{i} \in X}}\left(x_{2}\right) \cdots s_{e_{x_{i} \in X}}\left(x_{m}\right) s_{e_{y_{i} \in Y}}\left(y_{1}\right) s_{e_{y_{i} \in Y}}\left(\left(y_{2}\right)\right) \\
\cdots s_{e_{y_{i} \in Y}}\left(y_{n}\right) s_{e_{z_{i} \in Z}}\left(z_{1}\right) s_{e_{z_{i} \in Z}}\left(z_{2}\right) \cdots s_{e_{z_{i} \in Z}}\left(z_{u}\right)
\end{array}\right],
\end{aligned}
$$

\section{Method}

4.1. Method Overview. In this paper, we translate the traffic state recognition problem at the intersection into an image searching problem, and the imaged modeling of the intersection and the feature extraction of the image have been implemented in the sections above. This section presents an image searching method based on supervised hashing. The core idea of the method is to use the hash function to convert the image features into binary and then judge the similarity of the images by comparing the Hamming distance. In this way, it is only necessary to compare the image to be queried with the image with the label in the gallery to obtain the real content of the image to be inquired.

As shown in Figure 7, the architecture of traffic state recognition is proposed in this paper. Offline learning phase: Firstly, the method of the previous section is used to realize the image modeling at intersection and the feature extraction of the image. Secondly, the image feature vector is transformed into a fixed-length hash code by constructing a hash function. All images in the training library are hash coded to get a total hash change set. Online search phase: Firstly, the input data is imaged and feature extracted, and the data is hash coded by using the offline learning stage to obtain a hash function. Then, the Hamming distance of the hash code of the image to be checked, and the hash code of the image in the training library are compared. Finally, the query results are reordered according to the Hamming distance and returned, and the real content of the image to be inspected can be obtained.

\subsection{PCA Hashing Algorithm}

4.2.1. Objective Function. Similar to the existing study [42-44], we use PCA hash to solve the image searching problem. Basically, the PCA algorithm is used to reduce the original data. Then, a hash function is learnt to convert each data point of the image feature into a binary code.

The objective function can be formed by minimizing the target coding matrix and the resulting coding matrix as follows:

$$
\min _{H, W}\left\|H-W^{T} G\right\|_{F}^{2}+\rho \sum_{i=1}^{\varphi}\left\|h_{i}-\mu\right\|_{2}^{2}
$$

where $G \in R^{c * \varphi}$ represents a new matrix of the original data $E \in R^{\sigma * \varphi}$ after the dimensional reduction by the PCA and $G=T E \in R^{c * \varphi}, T \in R^{c * \sigma}$ represents the dimensionality reduction matrix. $W \in R^{c * c}$ represents the rotation matrix. $H=\left[h_{1}, h_{2}, \ldots, h_{\varphi}\right] \in R^{c * \varphi}$ is assumed to be a hashed coding matrix after quantization. $h_{i}$ is the hash code of each sample; $\mu=(1 / n) \sum_{i=1}^{n} h_{i}$ is the mean vector of all hash coded vectors. The main role of the regularization term $\sum_{i=1}^{\varphi}\left\|h_{i}-\mu\right\|_{2}^{2}$ is to reduce the influence of the outliers in the process of learning the hash function by reducing the deviation between the value of each hash code and the mean. $\rho$ is the weight of the penalty.

4.2.2. Algorithm. Equation (17) shows the objective function. Since the objective function is a convex function and is not smooth, it is difficult to directly find the optimal solution. Therefore, this paper uses a two-step alternating optimization method [45] to solve the problem.

(a) When $H$ remains constant, the problem becomes

$$
\min _{W}\left\|H-W^{T} G\right\|_{F}^{2}
$$




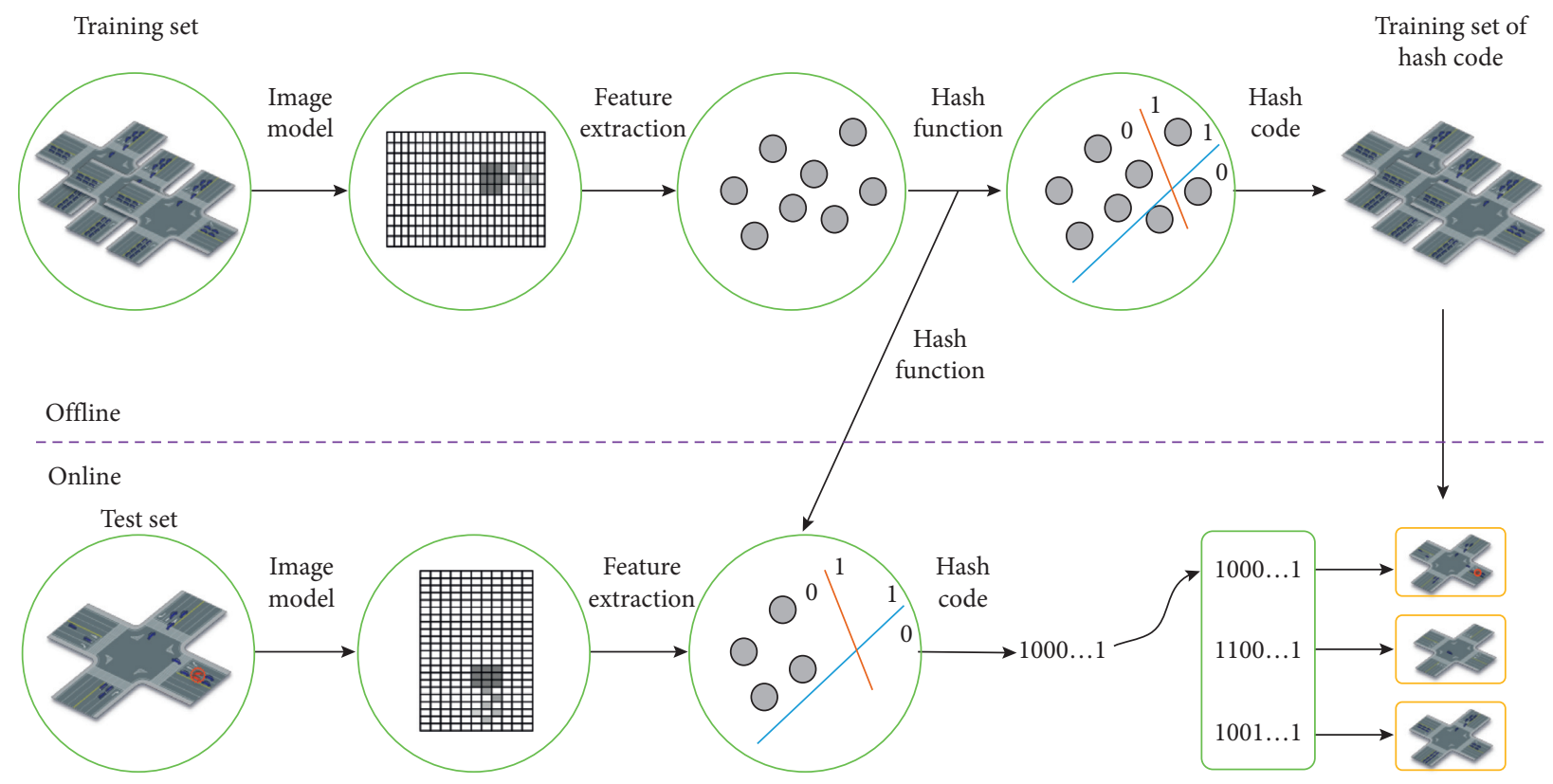

Figure 7: Traffic state recognition structure at the intersection.

Solution:

$$
W^{T}=H G^{-1} \text {. }
$$

(b) When $W$ remains constant, the problem becomes

$$
\min _{H}\left\|H-W^{T} G\right\|_{F}^{2}+\rho \sum_{i=1}^{\varphi}\left\|h_{i}-\mu\right\|_{2}^{2} .
$$

By mathematical derivation of equation (21), the result of $h_{i}$ is obtained:

$$
\begin{aligned}
\hat{h_{i}} & =\underset{H}{\arg \min }\left\|H-W^{T} G\right\|_{F}^{2}+\rho \sum_{i=1}^{\varphi}<h_{i}-\mu, h_{i}-\mu>, \\
& \left.=\underset{H}{\arg \min }\left\|H-W^{T} G\right\|_{F}^{2}+\rho \sum_{i=1}^{\varphi}\left(<h_{i}, h_{i}\right\rangle-2<h_{i}, \mu>+<\mu, \mu>\right), \\
& =\underset{H}{\arg \min }\left\|H-W^{T} G\right\|_{F}^{2}+\rho \sum_{i=1}^{\varphi}\left\|h_{i}\right\|_{2}^{2}-\rho \varphi\|\mu\|_{2}^{2} .
\end{aligned}
$$

By deriving equation (19) and letting the derivative be zero, we get the optimal solution of $h_{i}$ :

$$
\hat{h}_{i}=\frac{1}{1+\rho}\left(\left(W^{T} G\right)_{i}+\rho \frac{1}{\varphi} \sum_{i=1}^{\varphi}\left(W^{T} G\right)_{i}\right) .
$$

Binary coding for $h_{i}$ :

$$
\begin{aligned}
& b_{i(j)}=\operatorname{sgn}\left(h_{i(j)}\right)=\left\{\begin{array}{ll}
b_{i(j)}\left(h_{i(j)}\right)=1 & \text { if } h_{i(j)} \geq \mu(j) \\
b_{i(j)}\left(h_{i(j)}\right)=0 & \text { if } h_{i(j)}<\mu(j)
\end{array},\right. \\
& \mu(j)=\operatorname{mean}\left(h_{i(j)}\right), j=1,2, \ldots, \varphi .
\end{aligned}
$$

Finally, the hash code matrix $B=\left[b_{1}, b_{2}, \ldots, b_{\varphi}\right]$ of the original data set $E$ is obtained (PCA hashing algorithm is shown in Algorithm 1).

\section{Simulation Experiment and Case Study}

In this section, we present the (1) simulation experiment and (2) case study.

\subsection{Simulation Experiment}

5.1.1. VISSIM Simulation Environment. The signalized intersection is implemented in VISSIM. The detailed settings of the VISSIM simulation environment are listed in Table 2.

5.1.2. Training Set and Test Set. The sampling interval of the simulation data is set to $1 \mathrm{~s}$ to simulate the detection frequency of the advanced detector. This will give 36,000 samples. We used 30,000 samples as the training set and 6000 samples as the test set (see Table 3).

5.1.3. Analysis Results. The effects of fineness FI and effectiveness TM are evaluated.

$$
\left\{\begin{array}{l}
\mathrm{FI}=\frac{\mathrm{TP}}{\mathrm{TP}+\mathrm{FN}}, \\
\mathrm{TM}=\frac{\mathrm{FE}}{\mathrm{FT}},
\end{array}\right.
$$

where TP represents the number of samples that were correctly searched. FN indicates the number of samples that were incorrectly searched. FE represents the recognition time. FT represents the total search time. 
Input: training samples $E \in R^{\sigma * \varphi}$, control parameter $\rho$, number of dimensionality reduction $c$, random initialization $H=\left[h_{1}, h_{2}, \ldots, h_{\varphi}\right] \in R^{c * \varphi}$ and $W \in R^{c * c}$.

Output: hash coding matrix $B \in R^{c * \varphi}, T \in R^{c * \sigma}$ and $\mu$.

Process:

Start.

Using $G=T E \in R^{c * \varphi}$ to obtain $G$.

Using (12) to calculate $W$.

Using (13) to calculate $H$ and until convergence.

Using (15) to obtain hash function.

The binarization threshold vector is obtained by calculating the mean vector of all $H$ samples by $\operatorname{sgn}\left(h_{i(j)}\right)$.

Obtain a final hash coding matrix $B$ through $\mu$; Over.

Algorithm 1: PCA hashing algorithm.

TABLE 2: VISSIM simulation settings.

\begin{tabular}{|c|c|}
\hline Parameter & Value \\
\hline $\begin{array}{l}\text { Length of the connecting section of the } \\
\text { intersection }\end{array}$ & $470-490$ meters \\
\hline Lane width & 3.5 meters \\
\hline Number of lanes & 4 (two way) \\
\hline Turning ratio & $3: 7$ (turn left: straight) \\
\hline Speed distribution & {$[20 \mathrm{~km} / \mathrm{h}, 60 \mathrm{~km} / \mathrm{h}]$} \\
\hline Car type distribution & $3: 97$ (bus: car) \\
\hline Signal control & $\begin{array}{c}\text { Stage1-north and south straight. Stage2-north and south straight left. Stage3-east and west } \\
\text { straight. Stage4-east and west left. }\end{array}$ \\
\hline Stage time & Stage $1-40$ s. Stage2-20 s. Stage3-40 s. Stage4-20 s. \\
\hline Input flow & $\begin{array}{r}\text { Stage } 1-1000 \mathrm{v} / \mathrm{h} \text {. Stage } 2-500 \mathrm{~s} \text {. Stage } 3-1000 \mathrm{~s} \text {. Stage } 4-500 \mathrm{~s} \text {. Multiply the stage flow by a random } \\
\text { number } x \text { every } 3600 \mathrm{~s} .0 .5<x<2\end{array}$ \\
\hline Raw data & $\begin{array}{c}\text { Vehicle ID, vehicle speed, latitude and longitude (simulation), time stamp, vehicle length, vehicle } \\
\text { type, etc. }\end{array}$ \\
\hline Simulation time & $36000 \mathrm{~s}$ \\
\hline
\end{tabular}

TABle 3: Simulation data samples.

\begin{tabular}{lc}
\hline Type & Size \\
\hline Training set & 30000 \\
Test set & 6000 \\
\hline
\end{tabular}

This study is compared with the relevant research in the author's preliminary work [30,37]. From the comparison of results analysis, the method used in this paper has a higher improvement in the fineness and effectiveness of traffic state recognition at intersections (see Figure 8 , the data points are calculated once every 3600 s.). As shown in Figure 8(b), the reason for the large improvement in effectiveness is that the periodic recognition is used for the traffic state recognition at the intersection, and the real-time recognition is also used in the paper.

\subsection{Case Study}

5.2.1. Background. Balong Road intersection in Xuchang city is used as a case study. The calibration of static data is provided by the traffic management department, and the calibration of dynamic data is proceeded by using the detection data of wide-area radar, as shown in Figure 9.

5.2.2. Results and Discussion. The data is collected at weekdays' morning peak hours and the evening peak hours, as shown in Figure 10.

In this case study, the selected traffic states are undersaturated and oversaturated. The range of the undersaturated state is $\bar{s}_{1}=[0,1)$, and the range of the oversaturated state is $\bar{s}_{2}=[1,1.5)$.

As shown in Figure 11, the oversaturated occurred at the morning peak hours and the evening peak hours of Balong Road intersection. This judgment result is consistent with the change of traffic flow, as shown in Figure 10. Therefore, this case study proved the validity of the proposed method.

\section{Conclusions}

In this paper, inspired by the high-precision detection technology and image searching technology, we develop a new HTSI modeling method and a semisupervised hashbased TSI recognition algorithm. We present a detailed process of image modeling and an image searching method 


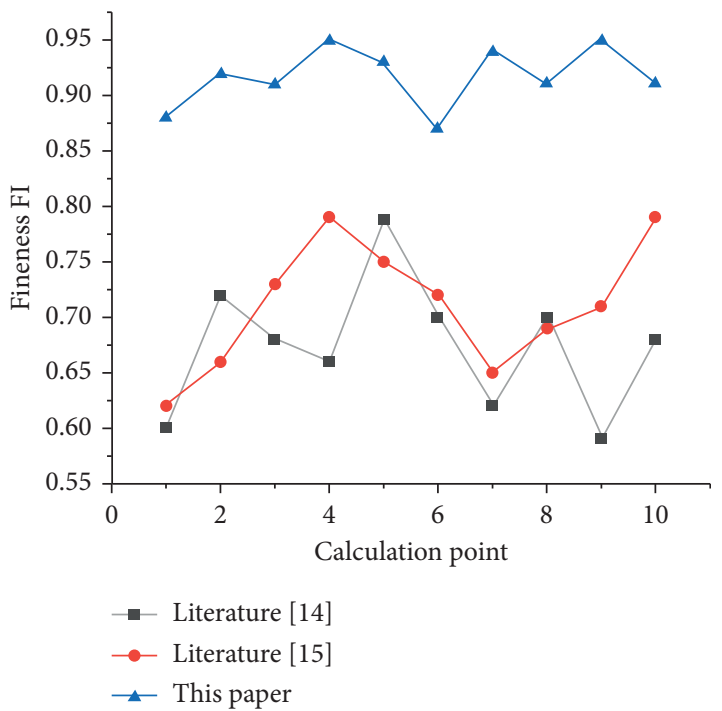

(a)

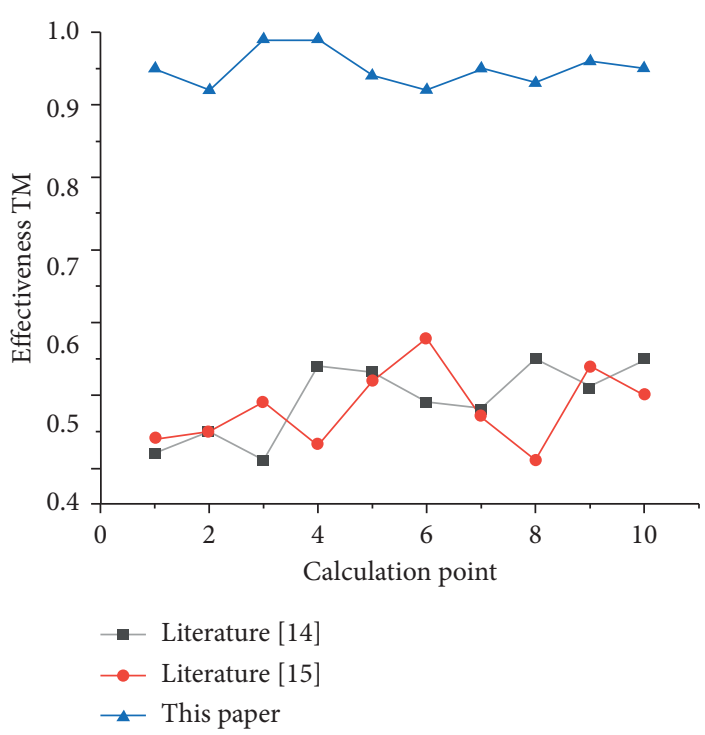

(b)

FIgURE 8: Traffic state recognition results of intersection. (a) Fineness comparison. (b) Effectiveness comparison.

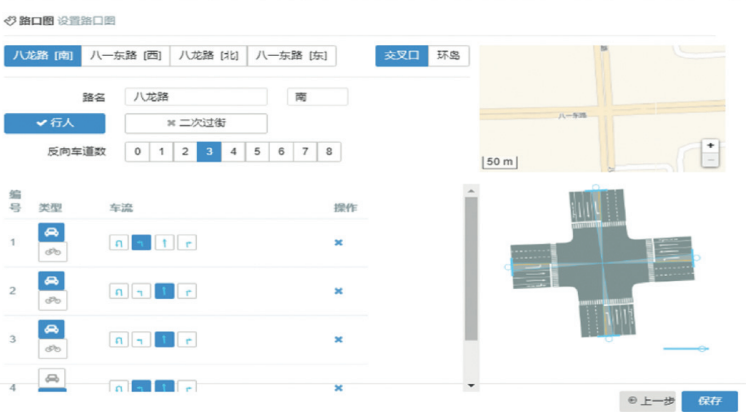

(a)

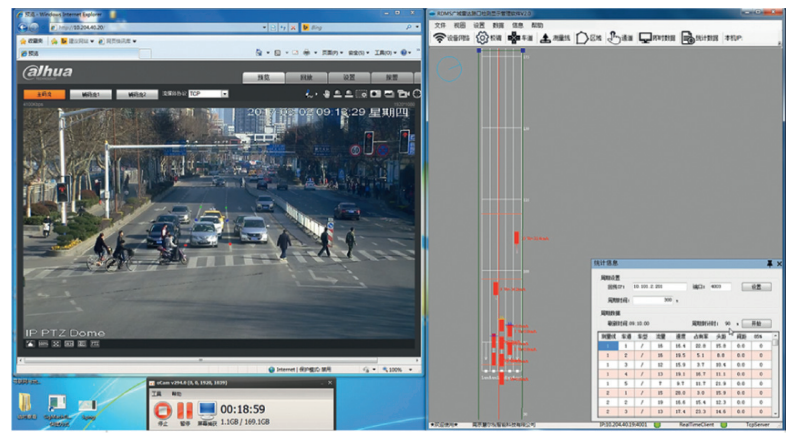

(c)

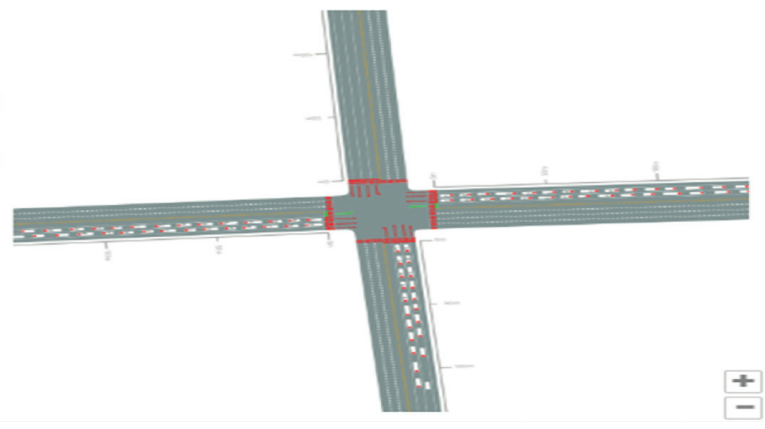

(b)

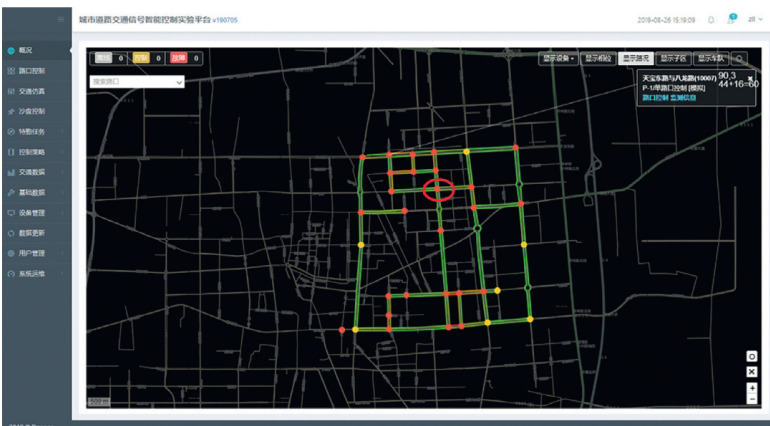

(d)

FIgURE 9: Balong road intersection in Xuchang city.

based on PCA hashing and carry out a comprehensive experiment by using the simulated data set. Results demonstrate that the proposed method is superior to the traditional traffic state recognition method in the fineness and effectiveness of state recognition. Furthermore, the potential of the proposed model is shown in the more complex mixed traffic state characteristics of intersections such as emergency vehicles, bus priority, and trams. However, there are still two shortcomings in this paper: (1) The applicability of intersections with mixed traffic without lane rules is ignored. (2) This paper requires that the accuracy of the detection data is sufficiently high, but does not consider the applicability in the case of insufficient data accuracy. In the future study, we will study the abovementioned two issues in detail and try to extend the method to short-term traffic flow estimation, traffic signal control, and other fields. 


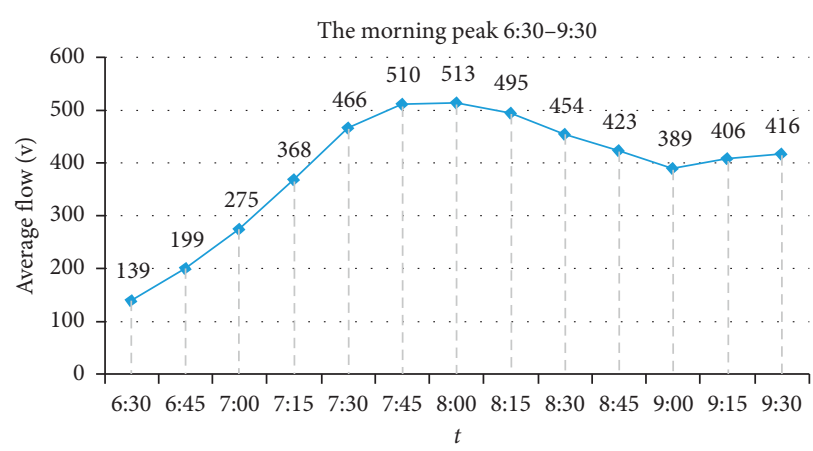

(a)

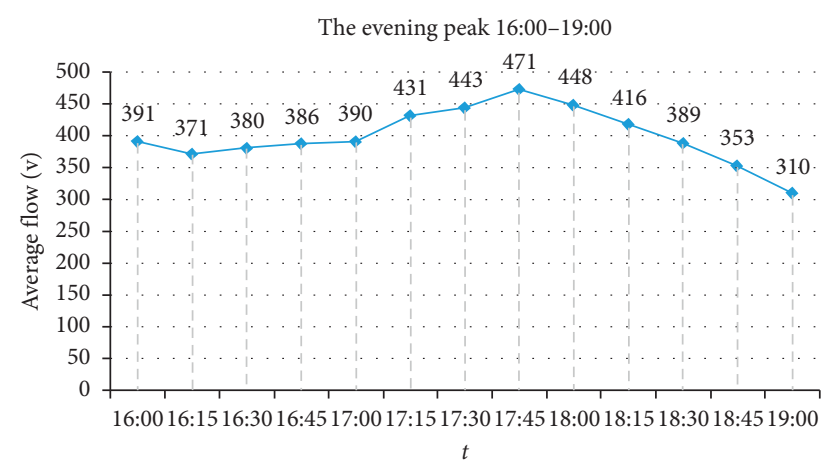

(b)

FIgURE 10: Morning and evening peak traffic flow of Balong road intersection.

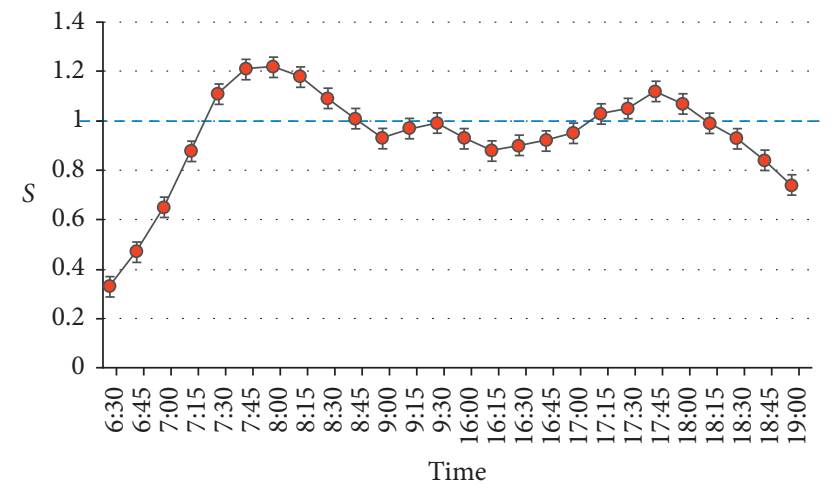

FIGURE 11: The morning and evening peak traffic status of Balong road intersection.

\section{Data Availability}

The data generated by the simulation are used in the paper, and the parameters have been calibrated in the simulation environment because the data are available. The data of the case in this paper are provided by Xuchang traffic police detachment in Henan Province. The data are the real traffic data of Xuchang City.

\section{Disclosure}

The views are those of the authors alone.

\section{Conflicts of Interest}

The authors declare no conflicts of interest.

\section{Authors' Contributions}

Li-li Zhang and Qi Zhao contributed equally to this work.

\section{Acknowledgments}

This research was supported by the Beijing Municipal Great Wall Scholar Program under grant no. CITTCD20190304, the National Key R\&D Program of China under grant nos. 2017YFC0821102 and 2017YFC0822504, the Basic Scientific Research Project of Beijing Municipal Education
Commission under grant no. 110052971803/013, and the North China University of Technology Youth Yuyou Program under grant no. 107051360019XN133/011.

\section{References}

[1] P. B. Hunt, D. I. Robertson, R. D. Bretherton, and M. C. Royle, "The SCOOT on-line traffic signal optimisation technique," Traffic Engineering \& Control, vol. 23, no. 4, 1982.

[2] J. Y. K. Luk, "Two traffic-responsive area traffic control methods: SCAT and SCOOT," Traffic Engineering \& Control, vol. 25, no. 1, 1984.

[3] P. Lowrie, SCATS-A Traffic Responsive Method of Controlling Urban Traffic, Roads and Traffic Authority, Sydney, New South Wales, Australia, 1990.

[4] G. Rafiq, B. Talha, M. Patzold et al., "What?s new in intelligent transportation systems?: an overview of European projects and initiatives," IEEE Vehicular Technology Magazine, vol. 8, no. 4, pp. 45-69, 2013.

[5] D. Green, P. Bennett, C. Han et al., "Cooperative intelligent transport systems (C-ITS) - an overview of the ARRB/Austroads C-ITS work program 2010-2013," World Journal of Diabetes, vol. 23, no. 2, pp. 15-28, 2014.

[6] I. Poupyrev, "Wide-field radar-based gesture recognition," U.S. Patent Application 16/153, 2019.

[7] X. Qu, T. Guo, J. Guo et al., "A real-time signal control strategy at an isolated pedestrian crossing based on radar data," Advances in Mechanical Engineering, vol. 11, no. 3, p. $1687814019825908,2019$.

[8] H. Tullberg, P. Popovski, D. Gozalvez-Serrano et al., "The METIS 5G system concept: meeting the 5G requirements," IEEE Communications Magazine, vol. 54, no. 12, pp. 132-139, 2015.

[9] A. Gupta and R. K. Jha, "A survey of 5G network: architecture and emerging technologies," IEEE Access, vol. 3, pp. 1206-1232, 2015.

[10] IMT-2020. 5G Concept[EB/OL]. http://www.imt-2020.org.cn/ $\mathrm{zh} /$ documents/listBy Query? currentPage=1\&content.

[11] T.-H. Chang and G.-Y. Sun, "Modeling and optimization of an oversaturated signalized network," Transportation Research Part B: Methodological, vol. 38, no. 8, pp. 687-707, 2004.

[12] H. Liu, K. N. Balke, and W.-H. Lin, “A reverse causal-effect modeling approach for signal control of an oversaturated intersection," Transportation Research Part C: Emerging Technologies, vol. 16, no. 6, pp. 742-754, 2008.

[13] H. Chai, R. Ma, and H. M. Zhang, "Search for parking: a dynamic parking and route guidance system for efficient 
parking and traffic management," Journal of Intelligent Transportation Systems, vol. 23, no. 6, pp. 541-556, 2019.

[14] N. Cui, B. Chen, K. Zhang, Y. Zhang, X. Liu, and J. Zhou, "Effects of route guidance strategies on traffic emissions in intelligent transportation systems," Physica A: Statistical Mechanics and Its Applications, vol. 513, pp. 32-44, 2019.

[15] M. Teichmann, M. Weber, M. Zoellner, R. Cipolla, and R. Urtasun, "Multinet: real-time joint semantic reasoning for autonomous driving," in Proceedings of the 2018 IEEE Intelligent Vehicles Symposium (IV), pp. 1013-1020, IEEE, Changshu, China, June 2018.

[16] J. Wang, J. Liu, and N. Kato, "Networking and communications in autonomous driving: a survey," IEEE Communications Surveys \& Tutorials, vol. 21, no. 2, pp. 1243-1274, 2018.

[17] C. Chen, A. Seff, A. Kornhauser, and J. Xiao, "Deepdriving: learning affordance for direct perception in autonomous driving," in Proceedings of the 2015 IEEE International Conference on Computer Vision (ICCV), pp. 2722-2730, IEEE, Santiago, Chile, December 2015.

[18] M. Grimnes and A. Aamodt, "A two layer case-based reasoning architecture for medical image understanding," Lecture Notes in Computer Science, Springer, Berlin, Heidelberg, pp. 164-178, 1996.

[19] A. C. Gallagher and T. Chen, "Understanding images of groups of people," in Proceeding of the 2009 IEEE conference on computer vision and pattern recognition, pp. 256-263, IEEE, Miami, FL, USA, June 2009.

[20] J. R. Bach, C. Fuller, A. Gupta et al., "Virage image search engine: an open framework for image management," International Society for Optics and Photonics, vol. 2670, pp. 76-87, 1996.

[21] H. Jegou, M. Douze, and C. Schmid, "Hamming embedding and weak geometric consistency for large scale image search," Lecture Notes in Computer Science, Springer, Berlin, Heidelberg, pp. 304-317, 2008.

[22] J. Wang, S. Kumar, and S. F. Chang, "Semi-supervised hashing for large-scale search," IEEE Transactions on Pattern Analysis and Machine Intelligence, vol. 34, no. 12, pp. 2393-2406, 2012.

[23] D. C. Gazis, "Optimum control of a system of oversaturated intersections," Operations Research, vol. 12, no. 6, pp. 815-831, 1964.

[24] H. X. Liu, X. Wu, W. Ma, and H. Hu, "Real-time queue length estimation for congested signalized intersections," Transportation Research Part C: Emerging Technologies, vol. 17, no. 4, pp. 412427, 2009.

[25] Z. Qian and J. M. Xu, "Recognition of oversaturated traffic conditions based on loop detection," Journal of South China University of Technology (Natural Science Edition), vol. 41, no. 8, pp. 93-98, 2013.

[26] X. Ban, P. Hao, and Z. Sun, "Real time queue length estimation for signalized intersections using travel times from mobile sensors," Transportation Research Part C, vol. 19, no. 6, pp. 1133-1156, 2011.

[27] F. Li, K. Tang, J. Yao, and K. Li, "Real-time queue length estimation for signalized intersections using vehicle trajectory data," Transportation Research Record: Journal of the Transportation Research Board, vol. 2623, no. 1, pp. 49-59, 2017.

[28] A. Wu and X. Yang, "Real-time Queue Length Estimation of Signalized Intersections Based on RFID Data," ProcediaSocial and Behavioral Sciences, vol. 96, pp. 1477-1485, 2013.

[29] S. H. Tang, X. M. Liu, and Z. M. Chen, "State discriminant and queue length estimation of an intersection based on video data," Road Traffic and Security, vol. 1, pp. 58-64, 2015.

[30] L. Wang, L. L. Zhang, M. Li et al., "Precise judging method study of intersection traffic state," Science Technology and Engineering, vol. 16, no. 3 4, pp. 290-295, 2016.
[31] C. Antoniou, H. N. Koutsopoulos, and G. Yannis, "Dynamic data-driven local traffic state estimation and prediction," Transportation Research Part C: Emerging Technologies, vol. 34, no. 9, pp. 89-107, 2013.

[32] Z. Wang, Q. Cai, B. Wu et al., "Cycle-by-Cycle queue length estimation for signalized intersections using multi-source data," Journal of Harbin Institute of Technology (New Series), vol. 22, no. 2, pp. 86-93, 2015.

[33] C. Ren, W. Zhang, L. Qin, and B. Sun, "Queue spillover management in a connected vehicle environment," Future Internet, vol. 10, no. 8, pp. 79-88, 2018.

[34] F. Dion, H. Rakha, and Y.-S. Kang, "Comparison of delay estimates at under-saturated and over-saturated pre-timed signalized intersections," Transportation Research Part B: Methodological, vol. 38, no. 2, pp. 99-122, 2004.

[35] J. Q. Nie and D. L. Xu, "A model for signal phase traffic status real-time discrimination based on occupancy," Command Control \& Simulation, vol. 35, no. 1, pp. 123-127, 2013.

[36] Z. M. Chen, X. M. Liu, W. X. Wu et al., "Recognition method of traffic state and its authenticity combined with signal control," Journal of Chongqing Jiaotong University(Natural Science), vol. 35, no. 6, pp. 95-100, 2016.

[37] L. L. Zhang, L. Wang, K. Pan et al., "Intersection traffic state distinguish method based on comprehensive projection," Journal of Transportation Systems Engineering and Information Technology, vol. 16, no. 2, pp. 83-91, 2016.

[38] P. Wang, H. Yu, W. Zhang et al., "Reak-time traffic status evaluation method for urban cooperative vehicle infrastructure system," China Journal of Highway and Transport, vol. 32, no. 6, pp. 176-187, 2019.

[39] Z. Y. Wu, X. Q. Ding, and C. X. Ju, "A method of discrete traffic state recognition based on deep learning," Journal of Transportation Systems Engineering and Information Technology, vol. 17, no. 5, pp. 129-136, 2017.

[40] W. Genders and S. Razavi, "Using a deep reinforcement learning agent for traffic signal control," 2016, https://arxiv. org/abs/1611.01142.

[41] C. W. Huang, B. Shuai, J. Zuo et al., "Correct entropy based operation performance evaluation about urban rail transportation non-networks system," Journal of Transportation Systems Engineering and Information Technology, vol. 16, no. 6, pp. 115-121, 2016.

[42] Y. J. Su, H. Xu, C. Lei et al., "PCA Hashing for image data retrieval," Application Research of Computers, vol. 35, no. 10, pp. 273-276, 2018.

[43] A. Abdullah, A. Andoni, R. Kannan, and R. Krauthgamer, "Spectral approaches to nearest neighbor search," in in Proceedings of the 2014 IEEE 55th Annual Symposium on Foundations of Computer Science (FOCS)., pp. 581-590, IEEE, Philadelphia, PA, USA, October 2014.

[44] W. Zhang, K. Gao, Y. Zhang et al., "Data-oriented locality sensitive hashing," in Proceedings of the 18th ACM International Conference on Multimedia, ACM, Firenze, Italy, pp. 1131-1134, 2010.

[45] X. Zhu, Q. Xie, Y. Zhu, X. Liu, and S. Zhang, "Multi-view multi-sparsity kernel reconstruction for multi-class image classification," Neurocomputing, vol. 169, no. 10, pp. 43-49, 2015. 